\title{
Chest X-ray and Uveitis Evaluation in a Population with Low Incidence of Sarcoidosis
}

Richard W. Yemm - Paula E. Pecen - Gregory D. Fliney •

Alan G. Palestine

Received: May 18, 2020 / Published online: July 1, 2020

(C) The Author(s) 2020

\section{ABSTRACT}

Introduction: Radiographic chest imaging is utilized in the workup of uveitis, with the purpose of diagnosing sarcoidosis. Sarcoidosis is an idiopathic systemic granulomatous inflammation which accounts for approximately $10 \%$ of uveitis. The prevalence of sarcoidosis and uveitis varies widely with geography and ethnicity. We studied the value of chest X-ray (CXR) in the evaluation of uveitis patients in the Western United States. The study was a retrospective chart review of 559 patients presenting to the Uveitis Department at University of Colorado between January 1, 2011 and July 31, 2017.

Methods: CXR and/or chest computerized tomography (CT) was obtained in patients with an unknown uveitic diagnosis. Presumed sarcoidosis was defined as the presence of bilateral

Digital Features To view digital features for this article go to https://doi.org/10.6084/m9.figshare.12464615.

R. W. Yemm · P. E. Pecen - G. D. Fliney ·

A. G. Palestine $(\bowtie)$

Department of Ophthalmology, University of

Colorado School of Medicine, Aurora, CO, USA

e-mail: alan.palestine@cuanschutz.edu hilar lymphadenopathy in a patient with uveitis. Age, race, and anatomic location of the uveitis were analyzed.

Results: The prevalence of sarcoidosis in our population was $4.3 \%$. The discovery rate of sarcoidosis by CXR was $2.6 \%$. Of 12 positive CXRs, 11 were in black patients. Our study identified five previously undiagnosed patients with sarcoidosis, four of whom were black.

Conclusions: CXR screening for sarcoid uveitis has a low yield in our population and is most likely to be positive in black patients. The prevalence of sarcoidosis is low in our study but not unlike the findings in similar demographic populations. Although chest CT scan is more sensitive than chest $\mathrm{X}$-ray, the cost and radiation dose are greater; thus CT may not be indicated in low incidence populations.

Keywords: Chest X-ray; CXR; Epidemiology; Sarcoidosis; Uveitis 


\section{Key Summary Points}

\section{Why carry out this study?}

The study was carried out to determine the utility of obtaining chest X-ray (CXR) in the workup of patients with uveitis.

The study aims to correlate pre-test probability with diagnostic yield of CXR in the context of demographic variables such as ethnicity.

\section{What was learned from the study?}

The incidence of sarcoidosis is low in our population and CXR was most likely to yield positive results in black patients.

Patient demographics should be taken into consideration when ordering laboratory and imaging tests in the workup of uveitis.

\section{INTRODUCTION}

Uveitis is a broad group of inflammatory diseases of the choroid, ciliary body, and/or iris, though nearly all ocular structures may be affected. It may be categorized by location (e.g., anterior, intermediate, posterior, pan), duration (e.g., acute, chronic, recurrent), and etiology (e.g., autoimmune, neoplastic, or infectious) [1].

The incidence of uveitis varies widely across different ethnic/racial groups and by geographic location, ranging from 17 to 52 per 100,000 people each year [2]. In a presumably comparable Western United States population (Northern California) to the population presented in our study, the incidence of uveitis is 52.4 per 100,000 person-years [3]. Despite its low incidence, uveitis causes $30 \%$ of new cases of legal blindness every year in the USA [4].

Sarcoidosis is a systemic granulomatous inflammatory disease with multiple ocular manifestations. In the eye it most often presents as anterior uveitis, with symptoms of eye pain, photophobia, and blurred vision, and exam findings of anterior chamber cell with granulomatous "mutton fat" keratoprecipitates. It is also known to cause posterior segment involvement including vitreous inflammation and granulomas of the choroid and optic nerve.

Systemic sarcoidosis is the leading cause of interstitial lung disease in developed countries [5]. It also affects the heart, liver, nervous system, lymphatics, and skin. About half of patients with systemic sarcoidosis will eventually develop ocular involvement [6]. Sarcoid uveitis may occur in the absence of other systemic disease, or may be the sentinel event that leads to the diagnosis of systemic disease.

Just as the epidemiology of uveitis varies across demographic groups, sarcoidosis varies to an even greater extent. Japan reports only 3.7 cases of sarcoidosis per 100,000 people per year, compared to 28.2 per 100,000 in Finland. While the incidence of sarcoidosis is low in Japan, the proportion of ocular involvement is rather high, ranging from $50 \%$ to $93 \%[7,8]$. In Sweden $0 \%$ of sarcoidosis cases are found in black patients, in contrast to the USA where black patients account for $66 \%$ of all sarcoidosis [6]. In the USA, black patients with sarcoidosis are 13.8 times more likely to have ocular involvement than all other racial/ethnic groups [9]. These differences are important to account for, especially in our patient population. Only $4.6 \%$ of the Colorado population is black, compared with $13.4 \%$ in the USA $[10,11]$.

The gold standard for diagnosis of sarcoidosis is tissue biopsy showing non-caseating granulomatous inflammation; however, ocular biopsy is rarely performed. Rather, a combination of clinical signs, laboratory testing, and imaging studies is used. Specific criteria were established in 2009 by the International Workshop on Ocular Sarcoidosis and revised in 2019 $[12,13]$.

Among the laboratory tests used in the workup of sarcoid uveitis are serum angiotensin-converting enzyme (ACE), serum lysozyme, and soluble serum interleukin-2 receptor. Standard chest imaging is by chest X-ray (CXR) but may also include chest computerized tomography (CT) in certain circumstances. The purpose of this study was to determine the 
utility of a screening CXR in patients with uveitis seen in the Department of Ophthalmology at the University of Colorado School of Medicine.

\section{METHODS}

A retrospective chart review was conducted for patients with uveitis seen at the University of Colorado Department of Ophthalmology by one of two uveitis specialists between January 1, 2011 and July 31, 2017. Standardization of uveitis nomenclature [12] was used to categorize uveitis by location and duration. The presence or absence of previously established etiology/ systemic disease was noted. Laboratory and imaging results were collected from 6 months before or after the initial visit with a uveitis specialist. This included serum ACE and lysozyme as well as CXR and chest CT results. Chest imaging was considered positive if bilateral hilar adenopathy was present. Syphilis serology was obtained on patients without a previously defined etiology. Colorado is not an endemic area for tuberculosis (TB) and the positive predictive value of TB skin testing and Quantiferon in patients with uveitis is $1 \%$ and $11 \%$, respectively [14]. Thus, TB testing was not routinely performed in our study. The Colorado Multiple Institutional Review Board granted approval for access to patient charts for the purposes of data collection as pertains to our study.

All statistics reported in the study were calculated using Microsoft Excel Version 16.37, 2020. Relative risk, sensitivity, and specificity were calculated via a chi-square test to analyze categorical variables.

\section{RESULTS}

Five hundred fifty-nine patients with uveitis presented for an initial visit between January 1, 2011 and July 31, 2017. The majority (58.3\%) were female. White patients comprised the largest percentage of the population $(44.3 \%)$, followed by black patients (15.7\%), Latinos $(12.5 \%)$, and Asians (2.5\%). Race was unable to be determined in $24.7 \%$. Almost half (49.2\%) of uveitis cases were categorized as anterior; $17.5 \%$ were intermediate, $16.8 \%$ were panuveitis, and $16.5 \%$ were posterior. One third (33\%) were acute (new onset) presentations of uveitis. The rest of the cases were chronic (45\%) or recurrent $(22 \%)$. The prevalence of sarcoidosis in our population was $4.3 \%$. Table 1 further delineates this data in patients with or without a previous diagnosis of sarcoidosis. Patients designated as having sarcoidosis either had a previous positive biopsy or met the International Workshop on Ocular Sarcoidosis (IWOS) 2019 criteria for presumed ocular sarcoidosis [13].

Of the 559 patients presenting with uveitis, 204 underwent CXR for evaluation of an undefined etiology for the inflammation. Patients with a defined uveitis syndrome and those with well-defined phenotypes, such as HLA-B27-associated anterior uveitis, did not receive chest imaging. Twelve CXRs revealed bilateral hilar adenopathy, five of which were previously undiagnosed cases of sarcoidosis. This yields a discovery rate of $2.6 \%$. As shown in Table 2, there were 12 positive CXRs, 11 of which were in black patients (this includes new and previously known diagnoses). Of the five new diagnoses of sarcoidosis made in our study, four were in black patients, yielding an 18-fold relative risk. Three of the newly diagnosed patients presented with anterior uveitis and two presented with intermediate uveitis. Two patients with history of biopsy-confirmed sarcoidosis had negative CXR.

Forty-one chest CT scans were performed, eight of which revealed bilateral hilar adenopathy. All eight patients with positive chest CTs had a previous positive CXR, but CT scan was recommended by the radiologist. Four of these positive CT scans were in patients with previous diagnoses of sarcoidosis. Six patients had both positive CXR and positive chest CT. Notably, our study did not identify any patients with a negative CXR and positive chest CT, nor were there any patients with positive CXR and negative chest CT. However, 19 patients with negative CXR underwent subsequent $\mathrm{CT}$ on the basis of the recommendation of the radiologist for uncertain findings on CXR. None of these patients were ultimately diagnosed with sarcoidosis or any other meaningful lung pathology. 
Table 1 Patient characteristics with and without sarcoidosis

\begin{tabular}{|c|c|c|c|}
\hline & Total population & Previously diagnosed sarcoidosis & No history of sarcoidosis \\
\hline Total & 559 & 19 & 540 \\
\hline \multicolumn{4}{|l|}{ Gender } \\
\hline Male & $231(41.3 \%)$ & $7(37 \%)$ & $224(41.5 \%)$ \\
\hline Female & $328(58.7 \%)$ & $12(63 \%)$ & $316(58.5 \%)$ \\
\hline \multicolumn{4}{|l|}{ Age } \\
\hline Average & 46.8 & 49.9 & 46.7 \\
\hline $0-39$ & $210(37.6 \%)$ & $5(26 \%)$ & $205(38.0 \%)$ \\
\hline $40-64$ & $228(40.8 \%)$ & $12(63 \%)$ & $216(40.0 \%)$ \\
\hline$>65$ & $121(21.6 \%)$ & $2(11 \%)$ & $119(22.0 \%)$ \\
\hline \multicolumn{4}{|l|}{ Race/ethnicity } \\
\hline White & $248(44.4 \%)$ & $4(21 \%)$ & $244(45.2 \%)$ \\
\hline Black & $87(15.6 \%)$ & $12(63 \%)$ & 75 (13.9\%) \\
\hline Hispanic & $72(12.9 \%)$ & $2(11 \%)$ & $70(13.0 \%)$ \\
\hline Asian & $14(2.5 \%)$ & $0(0 \%)$ & $14(2.6 \%)$ \\
\hline Unknown & $138(24.7 \%)$ & $1(5 \%)$ & $137(25.4 \%)$ \\
\hline \multicolumn{4}{|l|}{ Location of uveitis } \\
\hline Anterior & $275(49.2 \%)$ & $7(36.8 \%)$ & $268(49.6 \%)$ \\
\hline Intermediate & $98(17.5 \%)$ & $3(15.8 \%)$ & $95(17.6 \%)$ \\
\hline Posterior & $92(16.5 \%)$ & $1(5.3 \%)$ & $91(16.9 \%)$ \\
\hline Panuveitis & $94(16.8 \%)$ & $8(42.1 \%)$ & $86(15.9 \%)$ \\
\hline \multicolumn{4}{|l|}{ Chest X-ray } \\
\hline Total performed & 204 & 9 & 195 \\
\hline Positive & $12(5.9 \%)$ & $7(78 \%)$ & $5(2.6 \%)$ \\
\hline Negative & $192(94.1 \%)$ & $2(22 \%)$ & $190(97.4 \%)$ \\
\hline \multicolumn{4}{|l|}{ Chest CT } \\
\hline Total performed & 41 & 6 & 35 \\
\hline Positive & $8(19 \%)$ & $4(67 \%)$ & $4(11 \%)$ \\
\hline Negative & $33(81 \%)$ & $2(33 \%)$ & $31(89 \%)$ \\
\hline
\end{tabular}

Serum ACE was obtained in 199 patients. As shown in Table 3, 13 patients had elevated ACE; however, only six of these had sarcoidosis (including patients newly diagnosed in our study).
This yields a sensitivity of $31 \%$ and specificity of $96 \%$.

Serum lysozyme was obtained in 126 patients. Table 3 shows that 34 patients had 
Table 2 Patient characteristics associated with positive chest imaging

\begin{tabular}{|c|c|c|}
\hline & Positive chest $\mathrm{X}$-ray & Positive chest $\mathrm{CT}$ \\
\hline Total & 12 & 8 \\
\hline \multicolumn{3}{|l|}{ Gender } \\
\hline Male & $4(25 \%)$ & $2(25 \%)$ \\
\hline Female & $8(75 \%)$ & $6(75 \%)$ \\
\hline \multicolumn{3}{|l|}{ Age } \\
\hline Average & 52.6 & 57.4 \\
\hline \multicolumn{3}{|l|}{ Race/ethnicity } \\
\hline White & $1(8 \%)$ & $2(25 \%)$ \\
\hline Black & $11(92 \%)$ & $6(75 \%)$ \\
\hline Hispanic & 0 & 0 \\
\hline Asian & 0 & 0 \\
\hline Unknown & 0 & 0 \\
\hline \multicolumn{3}{|c|}{ Location of uveitis } \\
\hline Anterior & $6(50 \%)$ & $3(37.5 \%)$ \\
\hline Intermediate & $4(33 \%)$ & $3(37.5 \%)$ \\
\hline Posterior & 0 & 0 \\
\hline Panuveitis & $2(17 \%)$ & $2(25 \%)$ \\
\hline
\end{tabular}

elevated lysozyme, although only three of these patients had sarcoidosis. This yields a sensitivity of $60 \%$ and specificity of $74 \%$. Notably, positive predictive value is $9 \%$ and negative predictive value is $98 \%$.

\section{DISCUSSION}

Our study reveals several important trends regarding the workup of uveitis in the Western United States. Regional population differences affect the diagnostic yield of certain tests, with sarcoidosis being a prime example. Similarly, we did not perform routine $\mathrm{TB}$ testing in our patients because of low incidence in Colorado; however we believe it is appropriate to test for TB in select groups such as those with high exposure risk or those starting systemic immunosuppression. Only $4.5 \%$ of the Colorado population is black, yet black patients accounted for $15.7 \%$ of new uveitic presentations in our study. Despite representing a small portion of the population, $63 \%$ of patients with known sarcoidosis were black, and 11 of 12 patients with positive CXR were black. Additionally, four of the five patients with a new diagnosis of sarcoidosis were black.

We are unable to calculate the sensitivity or specificity of CXR or chest CT in our study because the majority of patients with previously diagnosed sarcoidosis did not undergo biopsy to confirm the diagnosis. Rather, diagnoses were made on the basis of positive chest imaging. Other studies have demonstrated these statistics, and the consensus is that chest CT is more

Table 3 Sensitivity and specificity of serum angiotensin-converting enzyme (ACE) and lysozyme

\begin{tabular}{cllllll}
\hline & Sarcoidosis & $\begin{array}{l}\text { No } \\
\text { sarcoidosis }\end{array}$ & Sensitivity & Specificity & $\begin{array}{l}\text { Positive predictive } \\
\text { value }\end{array}$ & $\begin{array}{l}\text { Negative predictive } \\
\text { value }\end{array}$ \\
\hline ACE & & & & & & \\
Total & 13 & 186 & $31 \%$ & $96 \%$ & $31 \%$ & $96 \%$ \\
Positive & $6(46 \%)$ & $7(3.8 \%)$ & & & & \\
Negative & $7(54 \%)$ & $179(96.2 \%)$ & & & $98 \%$ \\
Lysozyme & & & & & \\
Total & 5 & 121 & $60 \%$ & $74 \%$ & $9 \%$ & \\
Positive & $3(60 \%)$ & $31(25.6 \%)$ & & & & \\
Negative & $2(40 \%)$ & $90(74.4 \%)$ & & & & \\
\hline
\end{tabular}


sensitive at identifying sarcoid-related pulmonary changes. CT can identify subtle changes such as ground-glass opacities in stage 0 sarcoidosis before it is visible on CXR. While CXR is $90 \%$ sensitive for stage 1 pulmonary sarcoidosis, sensitivity falls when encompassing all stages [15].

In patients with uveitis, CXR has been shown to be $68 \%$ sensitive [16], while chest CT is $93 \%$ sensitive for diagnosing sarcoidosis [17]. In patients with a negative CXR but high clinical suspicion of sarcoid uveitis, chest CT is 73\% sensitive. The argument could be made that all patients with suspicion for sarcoid uveitis should undergo CT imaging rather than CXR. However, the monetary cost and radiation exposure make the topic controversial. Furthermore, we noted that 19 of the 204 patients in our study received a chest CT because of an equivocal finding on CXR and none of these patients were found to have any significant lung pathology. According to an online healthcare cost database [18], the in-network cost of a chest CT for an insured patient is 35 times higher than that of a CXR. Additionally, a single chest CT delivers 70 times the radiation of one CXR [19]. On the basis of this data, some physicians may consider screening with CXR and obtaining a chest CT only if negative, or instead being more selective in obtaining chest CT in the initial workup of uveitis. In our population this could mean a lower threshold for CT scan in black patients, with judicious use in other demographics. It should also be considered that a negative or indeterminate CXR may prompt a CT scan in some circumstances.

Serum ACE is $50 \%$ sensitive in our study which is consistent with prior studies on the sensitivity of ACE in diagnosing sarcoidosis $(41-72 \%)[20,21]$. However, when taking into account all patients with or without positive chest imaging, sensitivity of ACE falls to $31 \%$ while specificity is $96 \%$. Similarly, serum lysozyme had moderately low sensitivity in our study (60\%) but a high negative predictive value (98\%). This data suggests that serum ACE and lysozyme are poor screening tests but may be useful in ruling out the diagnosis of sarcoidosis in patients with uveitis. We did not obtain data regarding serum soluble interleukin-2 receptor levels because of the retrospective nature of our study, but more a recent study from 2017 has shown this to be useful [22].

The utility of establishing a diagnosis of sarcoidosis should be considered. Thirty to forty percent of uveitis cases reveal no specific cause and are determined to be idiopathic [23-25]. However, this does not preclude these patients from receiving adequate treatment. The treatment regimen for a given case of uveitis is often unaffected by the final diagnosis, or lack thereof. One particular study showed there was no significant difference in relapse rates between patients with an associated diagnosis and those with idiopathic uveitis [25]. Treatment of ocular sarcoidosis is not unlike certain other forms of uveitis, with the treatment regimen tailored to the extent and location of ocular inflammation. According to current pulmonology practices, systemic immunosuppressive therapy is not indicated for asymptomatic lung disease with stage 1 findings on $\mathrm{CXR}$, as the majority of these cases resolve without treatment $[9,26]$.

On the other hand, inadequately treated sarcoidosis may lead to morbidity and mortality. A 2015 study showed that cardiac conduction defects may be detectable on electrocardiogram in patients with asymptomatic sarcoidosis [27]. Han et al. [28] suggested that chest CT and electrocardiogram be obtained in all undiagnosed cases with high clinical suspicion for sarcoid uveitis.

Limitations of our study include a retrospective analysis and a low incidence of sarcoidrelated uveitis. Although chest CT may be more sensitive, our initial evaluation is, in general, CXR imaging. Additionally, a targeted approach to laboratory testing was done as outlined by others [14].

\section{CONCLUSION}

The incidence of sarcoidosis was low in our study population. This translated to a low rate of discovery by CXR, and hence the utility of CXR as a diagnostic test is less useful when the incidence of the disease is low. Black patients are more likely to develop uveitis and 
sarcoidosis than other demographic groups, and the yield for sarcoid screening in these patients is significantly higher.

\section{ACKNOWLEDGEMENTS}

Funding. This research was supported by a challenge grant from Research to Prevent Blindness, New York, NY. This support had no influence on the study design, interpretation, or reporting of the findings. No funding or sponsorship was received for the publication of this article.

Authorship. All named authors meet the International Committee of Medical Journal Editors (ICMJE) criteria for authorship for this article, take responsibility for the integrity of the work as a whole, and have given their approval for this version to be published.

Prior Presentation. Data from this manuscript was previously presented in poster form at ARVO 2019 in Vancouver, Canada.

Disclosures. Richard Yemm, Gregory Fliney, and Paula Pecen declare that they have no conflict of interest. Alan Palestine is a member of the journal's Editorial Board.

Compliance with Ethics Guidelines. The Colorado Multiple Institutional Review Board granted approval for access to patient charts for the purposes of data collection as pertains to our study.

Data Availability. The datasets generated during and/or analyzed during the current study are available from the corresponding author on reasonable request.

Open Access. This article is licensed under a Creative Commons Attribution-NonCommercial 4.0 International License, which permits any non-commercial use, sharing, adaptation, distribution and reproduction in any medium or format, as long as you give appropriate credit to the original author(s) and the source, provide a link to the Creative Commons licence, and indicate if changes were made. The images or other third party material in this article are included in the article's Creative Commons licence, unless indicated otherwise in a credit line to the material. If material is not included in the article's Creative Commons licence and your intended use is not permitted by statutory regulation or exceeds the permitted use, you will need to obtain permission directly from the copyright holder. To view a copy of this licence, visit http://creativecommons.org/licenses/by$\mathrm{nc} / 4.0 /$.

\section{REFERENCES}

1. Lopalco G, Venerito V, Sota J, et al. Epidemiological profile of non-infectious uveitis from the rheumatologist's perspective: a survey from two tertiary referral centres in Italy. Clin Exp Rheumatol. 2018;36(6 Suppl 115):68-37.

2. Jabs DA. Epidemiology of uveitis. Ophthalmic Epidemiol. 2008;15(5):283-4.

3. Gritz DC, Wong IG. Incidence and prevalence of uveitis in Northern California; the Northern California Epidemiology of Uveitis Study. Ophthalmology. 2004;111(3):491-500.

4. Nussenblatt RB. The natural history of uveitis. Int Ophthalmol. 1990;14(5-6):303-8.

5. Demedts M, Wells AU, Antó JM, et al. Interstitial lung diseases: an epidemiological overview. Eur Respir J. 2001;18(32):2-16.

6. Jones N, Mochizuki M. Sarcoidosis: epidemiology and clinical features. Ocul Immunol Inflamm. 2010;18(2):72-9.

7. Ohara K, Okubo A, Sasaki H, et al. Intraocular manifestations of systemic sarcoidosis. Jpn J Ophthalmol. 1992;36:452-7.

8. Matsuo T, Fujiwara N, Nakata Y. First presenting signs or symptoms of sarcoidosis in a Japanese population. Jpn J Ophthalmol. 2005;49:149-52.

9. Baughman RP, Teirstein AS, Judson MA, et al. Clinical characteristics of patients in case control study of sarcoidosis. Am J Respir Crit Care Med. 2001;164:1885-9. 
10. United States Census Bureau. Quick Facts: Colorado. https://www.census.gov/quickfacts/CO. Accessed 22 Jul 2019.

11. United States Census Bureau. Quick Facts: United States. https://www.census.gov/quickfacts/fact/ table/US/PST045218. Accessed 22 Jul 2019.

12. Herbort CP, Rao NA, Mochizuki M, et al. International criteria for the diagnosis of ocular sarcoidosis: results of the first International Workshop on Ocular Sarcoidosis (IWOS). Ocul Immunol Inflamm. 2009;17(3):160-9.

13. Mochizuki M, Smith JR, Takase H, et al. Revised criteria of International Workshop on Ocular Sarcoidosis (IWOS) for the diagnosis of ocular sarcoidosis. Br J Ophthalmol. 2019;103(10):1418-22.

14. Jabs DA, Busingye J. Approach to the diagnosis of the uveitides. Am J Ophthalmol. 2013;156(2): 228-36.

15. Greco FG, Spagnolo P, Muri M, et al. The value of chest radiograph and computed tomography in pulmonary sarcoidosis. Sarcoidosis Vasc Diffuse Lung Dis. 2014;31(2):108-16.

16. Acharya NR, Browne EN, Rao N, Mochizuki M. Distinguishing features of ocular sarcoidosis in an international cohort of uveitis patients. Ophthalmology. 2018;125(1):119-26.

17. Kaiser PK, Lowder CY, Sullivan P, et al. Chest computerized tomography in the evaluation of uveitis in elderly women. Am J Ophthalmol. 2002;133(4):499-505.

18. Fair Health Consumer. Medical costs. https://www. fairhealthconsumer.org/medical. Accessed $22 \mathrm{Jul}$ 2019.

19. Mettler FA, Huda W, Yoshizumi TT, Mahesh M. Effective doses in radiology and diagnostic nuclear medicine: a catalog. Radiology. 2008;248(1): 254-63.
20. Ungprasert P, Carmona EM, Crowson CS, Matteson EL. Diagnostic utility of angiotensin-converting enzyme in sarcoidosis: a population-based study. Lung. 2016;194:91-5.

21. Niederer RL, Al-Janabi A, Lightman SL, TomkinsNetzer O. Serum angiotensin-converting enzyme has a high negative predictive value in the investigation for systemic sarcoidosis. Am J Ophthalmol. 2018;194:82-7.

22. Nguyen CTH, Kambe N, Kishimoto I, Ueda-Hayakawa I, Okamoto H. Serum soluble interleukin-2 receptor level is more sensitive than angiotensinconverting enzyme or lysozyme for diagnosis of sarcoidosis and may be a marker of multiple organ involvement. J Dermatol. 2017;44(7):789-97.

23. Bodaghi B, Cassoux N, Wechsler B, et al. Chronic severe uveitis: etiology and visual outcome in 927 patients from a single center. Medicine. 2001;80(4): 263-70.

24. Barisani-Asenbauer T, Maca SM, Mejdoubi L, Emminger W, Machold K, Auer H. Uveitis-a rare disease often associated with systemic diseases and infections-a systematic review of 2619 patients. Orphanet J Rare Dis. 2012;7:57.

25. Hadjadj J, Dechartres A, Chapron T, et al. Relevance of diagnostic investigations in patients with uveitis: retrospective cohort study on 300 patients. Autoimmun Rev. 2017;16(5):504-11.

26. Iannuzzi MC, Fontana JR. Sarcoidosis: clinical presentation, immunopathogenesis, and therapeutics. JAMA. 2001;305(4):391-9.

27. Kandolin R, Lehtonen J, Airaksinen J. Cardiac sarcoidosis: epidemiology, characteristics, and outcome over 25 years in a nationwide study. Circulation. 2015;131(7):624-32.

28. Han YS, Rivera-Grana E, Salek S, Rosenbaum JT. Distinguishing uveitis secondary to sarcoidosis from idiopathic disease: cardiac implications. JAMA Ophthalmol. 2018;136(2):109-15. 